\title{
An analytic approach for computing pi-coefficients of continuous-time systems
}

\author{
Shao-Chung Hu, I-Kong Fong* \\ Department of Electrical Engineering, National Taiwan University, Taipei, Taiwan 10617, ROC \\ Received 2 April 1996; received in revised form 4 December 1996; accepted 2 April 1997
}

\begin{abstract}
The pi-sharing theory for continuous-time systems is discussed in this paper. An analytic approach is proposed for computing pi-coefficients of LTI systems. For the two scalar pi-coefficients, feasible regions are characterized graphically on a plane, and for the other two matrix pi-coefficients, the corresponding values are obtained by solving a Riccati equation. The results are applied in an example to determine the stability of a feedback system containing both linear and nonlinear subsystems. (1) 1997 Elsevier Science B.V.
\end{abstract}

Keywords: Pi-sharing theory; Nonlinear system; Stability; Riccati equation; Hysteresis

\section{Introduction}

The stability analysis problem for systems with nonlinear components has attracted the attention of control theorists and engineers for a long time. There are not only many different analysis methods, but also many different definitions of stability of such systems. In [6,7], Lawrence and Johnson combine the state and inputoutput stability measures to establish the so-called pi-sharing theory. Like other existing methods, this theory cannot solve all problems, but it does provide an alternative approach. In pi-sharing theory, a set of picoefficients $\{\boldsymbol{\Gamma}, \boldsymbol{Q}, p, r\}$ is used to represent system energy exchange properties. Various stability definitions, such as Lyapunov stability, passivity, and finite mean square gain (FMSG) stability can be described by these coefficients. Thus, the theory has the potential for solving a wider class of stability analysis problems. However, because pi-coefficients for a system are not unique, it is not easy to decide all possible candidates directly from the definition. Furthermore, an inappropriate choice may make the whole analysis too conservative, or even useless.

Based on properties of composite pi-coefficients for feedback systems, $\mathrm{Wu}$ et al. [9] formulate the max-p problem, in which one of the pi-coefficients, $p$, describing the output energy dissipation rate of a system, is suggested to be maximized to obtain the largest FMSG stability range. With this formulation, a set of pi-coefficients of second-order discrete-time systems are derived and applied to analyze the FMSG stability of some adaptive systems [2]. Today, it can be recognized that the max- $p$ problem is actually a linear matrix inequality optimization problem [1], which is convex and can be numerically solved quite efficiently.

*Corresponding author. Fax: 886-2-3660449; e-mail: ikfong@cc.ee.ntu.edu.tw. 
In this paper, an analytic approach is presented for computing ranges of feasible pi-coefficients for a given continuous-time system. The results can be conveniently represented on the $r-p$ plane, where feasible regions of $r$ and $p$ can be shown graphically, and $\Gamma$ and $Q$ can be solved corresponding to every feasible pair of $r$ and $p$. With this approach, the pi-sharing theory can be applied more flexibly, and the associated stability problem can be analyzed more completely. To illustrate this point, we consider the stability analysis problem of a feedback system with linear subsystem in the feedforward path and a hysteresis relay in the feedback path. It is shown that the pi-sharing theory can give a simple sufficient condition for the stability of this system.

Below we introduce some notations to be used. A positive- (negative)-definite symmetric matrix $\boldsymbol{S}$ is denoted as $\boldsymbol{S}>(<) \mathbf{0}$. Similar notations are used for semi-definite matrices. Furthermore, $\boldsymbol{S}_{1}>\boldsymbol{S}_{2}$ means $\boldsymbol{S}_{1}-\boldsymbol{S}_{2}>\mathbf{0}$, etc. For a vector $\boldsymbol{v},|\boldsymbol{v}|=\sqrt{\boldsymbol{v}^{\mathrm{T}} \boldsymbol{v}}$. For a real function of time $f(\cdot),\|f\|_{T}=\left\{\int_{0}^{T} f^{2}(t) \mathrm{d} t\right\}^{1 / 2}$.

\section{Pi-sharing theory}

First, we establish some basic results regarding pi-sharing theory of single-input-single-output (SISO) continuous-time systems in state-space form:

$$
\begin{aligned}
& \dot{x}(t)=A(t) x(t)+b(t) u(t), \\
& y(t)=c^{\mathrm{T}}(t) x(t)+d(t) u(t),
\end{aligned}
$$

where $\boldsymbol{x}(t) \in \mathbb{R}^{n}, u(t) \in \mathbb{R}, y(t) \in \mathbb{R}$, and $\boldsymbol{A}, \boldsymbol{b}, \boldsymbol{c}, \boldsymbol{d}$ are system matrices with appropriate dimensions. Following [7], we say the system (1) is pi-sharing with respect to pi-coefficients $\{\Gamma(t), Q(t), p(t), r(t)\}$ if for all $T \geqslant 0$,

$$
\begin{aligned}
\int_{0}^{T} u(t) y(t) \mathrm{d} t \geqslant & \boldsymbol{x}^{\mathrm{T}}(T) \Gamma(T) \boldsymbol{x}(T)-\boldsymbol{x}^{\mathrm{T}}(0) \boldsymbol{\Gamma}(0) \boldsymbol{x}(0)+\int_{0}^{T} \boldsymbol{x}^{\mathrm{T}}(t) \boldsymbol{Q}(t) \boldsymbol{x}(t) \mathrm{d} t \\
& +\int_{0}^{T} p(t) y^{2}(t) \mathrm{d} t+\int_{0}^{T} r(t) u^{2}(t) \mathrm{d} t
\end{aligned}
$$

where both $\Gamma(t) \geqslant 0$ and $Q(t) \geqslant 0$ for all $t \in[0, T]$. The physical meanings of pi-coefficients can be found in [7]. For example, if $p(t)>0$ or $r(t)>0$, then the system "dissipates energy", otherwise it "generates energy" and tends to be destabilizing.

With (1) substituted in, (2) can be re-written as

$$
\begin{gathered}
\boldsymbol{x}^{\mathrm{T}}(T) \Gamma(T) \boldsymbol{x}(T)-\boldsymbol{x}^{\mathrm{T}}(0) \Gamma(0) \boldsymbol{x}(0)+\int_{0}^{T} \boldsymbol{x}^{\mathrm{T}}(t) \boldsymbol{Q}(t) \boldsymbol{x}(t) \mathrm{d} t+\int_{0}^{T} p(t) y^{2}(t) \mathrm{d} t \\
\quad+\int_{0}^{T} r(t) u^{2}(t) \mathrm{d} t-\int_{0}^{T} u(t) y(t) \mathrm{d} t=\int_{0}^{T}\left[\boldsymbol{x}^{\mathrm{T}}(t) u(t)\right] \boldsymbol{M}(t)\left[\boldsymbol{x}^{\mathrm{T}}(t) u(t)\right]^{\mathrm{T}} \mathrm{d} t \leqslant 0,
\end{gathered}
$$

where

$$
\begin{aligned}
& \boldsymbol{M}(t)=\left[\begin{array}{ll}
\boldsymbol{M}_{1}(t) & \boldsymbol{m}_{2}(t) \\
\boldsymbol{m}_{2}^{\mathrm{T}}(t) & M_{4}(t)
\end{array}\right] \\
& \boldsymbol{M}_{1}(t)=\boldsymbol{A}^{\mathrm{T}}(t) \Gamma(t)+\Gamma(t) \boldsymbol{A}(t)+\dot{\Gamma}(t)+\boldsymbol{Q}(t)+p(t) \boldsymbol{c}(t) \boldsymbol{c}^{\mathrm{T}}(t) \\
& \boldsymbol{m}_{2}(t)=\boldsymbol{\Gamma}(t) \boldsymbol{b}(t)-\frac{1}{2} \boldsymbol{c}(t)+p(t) d(t) \boldsymbol{c}(t) \\
& M_{4}(t)=-d(t)+r(t)+p(t) d^{2}(t)
\end{aligned}
$$




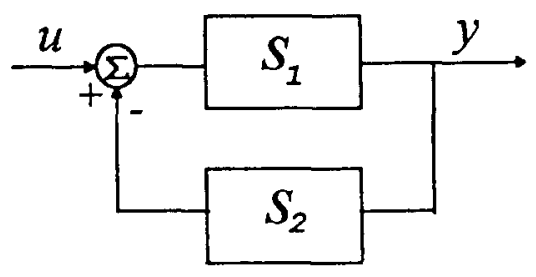

Fig. 1. A feedback system.

The matrix $\boldsymbol{M}(t)$ is called the dissipativity matrix of the system (1), with which we can state the following lemma for pi-coefficients in terms of its negative semi-definiteness:

Lemma 1 (Lawrence [6]). If for all $t, \boldsymbol{M}(t) \leqslant \mathbf{0}$ for some $\Gamma(t) \geqslant \mathbf{0}$ and $Q(t) \geqslant 0$, then (1) is pi-sharing with respect to $\{\boldsymbol{\Gamma}(t), \boldsymbol{Q}(t), p(t), r(t)\}$.

Combining the input-output stability and the state-space stability, Lawrence and Johnson [7] propose the so-called pi-stability for discrete-time systems. Here we can state its continuous-time version as follows:

Definition 1. The system (1) is said to be pi-stable, if there exist $\gamma_{1}, \gamma_{2}, \gamma_{3}, \gamma_{4} \in \mathbb{R}$ such that

$$
\|y\|_{T} \leqslant \gamma_{1}\|u\|_{T}+\gamma_{2}\left|x_{0}\right|
$$

and

$$
\sup _{0 \leqslant t \leqslant T}|x(t)| \leqslant \gamma_{3}\|u\|_{T}+\gamma_{4}\left|x_{0}\right|, \quad \forall u(t) \in \mathbb{R}, \quad x_{0} \in \mathbb{R}^{n}, \quad T \geqslant 0 .
$$

Similar to the case of discrete-time systems, we can establish many facts about pi-coefficients and their relationship to pi-stability. The results are summarized in the following lemmas, for which the proofs are parallel to their discrete-time counterparts, and thus omitted.

Lemma 2 (Lawrence [6]). If there exist $r_{0} \in \mathbb{R}, p_{0}>0$ and $\gamma>0$ such that $r(t) \geqslant r_{0}, p(t) \geqslant p_{0}$ and $\Gamma(t) \geqslant \gamma \boldsymbol{I}$ for all $t$, then the system (1) is pi-stable.

For the feedback system shown in Fig. 1, the composite pi-coefficients can be obtained from pi-coefficients of the subsystems.

Lemma 3 (Lawrence [6]). Let subsystems $S_{1}$ and $S_{2}$ be pi-sharing with respect to $\left\{\Gamma_{1}(t), Q_{1}(t), p_{1}(t), r_{1}(t)\right\}$ and $\left\{\boldsymbol{\Gamma}_{2}(t), \boldsymbol{Q}_{2}(t), p_{2}(t), r_{2}(t)\right\}$, respectively, and let $S$ be the system resulting from the feedback connection of $S_{1}$ and $S_{2}$. If $r_{1}(t), r_{2}(t), p_{1}(t)$, and $p_{2}(t)$ are finite and $\inf _{t \geqslant 0}\left\{r_{1}(t)+p_{2}(t)\right\}>0$, then $S$ is pi-sharing with respect to

$$
\{\boldsymbol{\Gamma}(t), \boldsymbol{Q}(t), p(t), r(t)\}=\left\{\left[\begin{array}{cc}
\Gamma_{1}(t) & \mathbf{0} \\
\mathbf{0} & \boldsymbol{\Gamma}_{2}(t)
\end{array}\right],\left[\begin{array}{cc}
\boldsymbol{Q}_{1}(t) & \mathbf{0} \\
\mathbf{0} & \boldsymbol{Q}_{2}(t)
\end{array}\right], p_{1}(t)+r_{2}(t), \frac{r_{1}(t) p_{2}(t)}{r_{1}(t)+p_{2}(t)}\right\},
$$

which are called the composite pi-coefficients for the feedback system $S$.

Thus, a sufficient condition to ensure that $S$ is pi-stable can be stated:

Theorem 1 (Lawrence [6]). For the feedback system $S$ shown in Fig. 1, suppose subsystems $S_{1}$ and $S_{2}$ are pi-sharing with respect to $\left\{\boldsymbol{\Gamma}_{1}(t), \boldsymbol{Q}_{1}(t), p_{1}(t), \boldsymbol{r}_{1}(t)\right\}$ and $\left\{\boldsymbol{\Gamma}_{2}(t), \boldsymbol{Q}_{2}(t), p_{2}(t), \boldsymbol{r}_{2}(t)\right\}$, respectively. Then the system $S$ is pi-stable, provided the following conditions are satisfied:

(i) $\exists \gamma_{1}>0$ and $\gamma_{2}>0$ such that $\boldsymbol{\Gamma}_{1}(t) \geqslant \gamma_{1} I$ and $\boldsymbol{\Gamma}_{2}(t) \geqslant \gamma_{2} I$ for all $t \geqslant 0$; 
(ii) $p_{1}(t), r_{1}(t), p_{2}(t), r_{2}(t)$ are finite, and

$$
\inf _{t \geqslant 0}\left\{r_{1}(t)+p_{2}(t)\right\}>0, \quad \inf _{t \geqslant 0}\left\{p_{1}(t)+r_{2}(t)\right\}>0 .
$$

This result shows the characteristic of energy exchange between two subsystems: if one subsystem generates energy $\left(p_{1}<0\right.$ or $\left.r_{1}<0\right)$, but the other subsystem dissipates more energy $\left(r_{2}>0\right.$ or $\left.p_{2}>0\right)$, then the whole system can remain stable (dissipate energy). Thus, if the individual subsystem's pi-coefficients can be derived, then the stability analysis problem of composite feedback system can be handled with ease.

However, to derive a useful set of pi-coefficients is in general not so easy. In [6], Lawrence considers the problem for discrete-time systems under quite restrictive conditions. Wu et al. [9] formulate the max- $p$ problem to look for pi-coefficients with certain optimality nature. Subsequently, it is shown [3] that to reach the optimality conditions the resulting formulas for pi-coefficients can be very complex, especially for highorder systems. If analytic solutions are not needed, then efficient numerical methods exist for solving the max-p problem, as it is a linear matrix inequality optimization problem [1].

\section{Main results}

In this paper, the primary goal is to find a more explicit way of handling general order LTI continuous-time systems. The emphasis is on the characterization of ranges of usable pi-coefficients for a system, and not on the derivation of a particular set of pi-coefficients. With the characterization, stability of feedback systems can be analyzed more completely.

To simplify the problem, we confine our attention to the time-invariant case. In addition to assuming that (1) is time-invariant, we also assume that only time-invariant pi-coefficients are looked for. Moreover, it is assumed that (1) is both controllable and observable. We start from Lemma 1, which requires that $\boldsymbol{M} \leqslant \mathbf{0}$. Since $\boldsymbol{M}$ can be partitioned in the form of (3), if $M_{4}=-d+r+p d^{2}<0$ and $\boldsymbol{M}_{1}-\boldsymbol{m}_{2} M_{4}^{-1} \boldsymbol{m}_{2}^{\mathrm{T}} \leqslant \mathbf{0}$, then $\boldsymbol{M} \leqslant \mathbf{0}$ [5]. Expanding $\boldsymbol{M}_{1}-\boldsymbol{m}_{2} M_{4}^{-1} \boldsymbol{m}_{2}^{\mathrm{T}} \leqslant \mathbf{0}$ with (3), we have

$$
\boldsymbol{A}^{\mathrm{T}} \boldsymbol{\Gamma}+\boldsymbol{\Gamma} \boldsymbol{A}+\boldsymbol{Q}+p \boldsymbol{c} \boldsymbol{c}^{\mathrm{T}}-\left[\boldsymbol{\Gamma} \boldsymbol{b}+\left(p d-\frac{1}{2}\right) \boldsymbol{c}\right]\left(-d+r+p d^{2}\right)^{-1}\left[\boldsymbol{b}^{\mathrm{T}} \boldsymbol{\Gamma}+\left(p d-\frac{1}{2}\right) \boldsymbol{c}^{\mathrm{T}}\right] \leqslant \mathbf{0} .
$$

This is equivalent to

$$
\overline{\boldsymbol{A}}^{\mathrm{T}} \boldsymbol{\Gamma}+\boldsymbol{\Gamma} \overline{\boldsymbol{A}}+\boldsymbol{\Gamma} \overline{\boldsymbol{R}} \boldsymbol{\Gamma}+\overline{\boldsymbol{Q}} \leqslant \mathbf{0},
$$

where

$$
\begin{aligned}
\overline{\boldsymbol{A}} & =\boldsymbol{A}-\left(-d+r+p d^{2}\right)^{-1}\left(p d-\frac{1}{2}\right) \boldsymbol{b} \boldsymbol{c}^{\mathrm{T}}, \\
\overline{\boldsymbol{R}} & =-\left(-d+r+p d^{2}\right)^{-1} \boldsymbol{b} \boldsymbol{b}^{\mathrm{T}}, \\
\overline{\boldsymbol{Q}} & =\boldsymbol{Q}+\left[p-\left(p d-\frac{1}{2}\right)^{2}\left(-d+r+p d^{2}\right)^{-1}\right] \boldsymbol{c} \boldsymbol{c}^{\mathrm{T}} \\
& =\boldsymbol{Q}+\left(p r-\frac{1}{4}\right)\left(-d+r+p d^{2}\right)^{-1} \boldsymbol{c} \boldsymbol{c}^{\mathrm{T}} .
\end{aligned}
$$

The equality part of (5) is a Riccati equation

$$
\overline{\boldsymbol{A}}^{\mathrm{T}} \boldsymbol{\Gamma}+\boldsymbol{\Gamma} \overline{\boldsymbol{A}}+\boldsymbol{\Gamma} \overline{\boldsymbol{R}} \boldsymbol{\Gamma}+\overline{\boldsymbol{Q}}=\mathbf{0}
$$

with Hamiltonian matrix

$$
\boldsymbol{H}=\left[\begin{array}{cc}
\bar{A} & \overline{\boldsymbol{R}} \\
-\overline{\boldsymbol{Q}} & -\overline{\boldsymbol{A}}^{\mathrm{T}}
\end{array}\right]
$$


Thus, the three lemmas below can be used to guarantee that the Riccati equation (9) has solutions and the inequality (5) has positive-definite solutions.

Lemma 4 (Knobloch [4]). If $\overline{\boldsymbol{R}} \geqslant \mathbf{0}$ and the pair $(\overline{\boldsymbol{A}}, \overline{\boldsymbol{R}})$ is controllable, then the following two properties are equivalent:

(i) $\boldsymbol{H}$ has no eigenvalues on the imaginary axis.

(ii) The Riccati equation (9) has a unique symmetric solution $\boldsymbol{\Gamma}^{-}$, such that all eigenvalues of $\overline{\boldsymbol{A}}+\overline{\boldsymbol{R}} \boldsymbol{\Gamma}^{-}$ are in the open lefi-half plane (OLHP).

Lemma 5 (Knobloch [4]). Suppose $\overline{\boldsymbol{R}} \geqslant \mathbf{0}$ and $\overline{\boldsymbol{Q}} \geqslant \mathbf{0}$. Moreover, suppose $\overline{\boldsymbol{A}}$ has all eigenvalues in the $O L H P$, then any symmetric solution $\Gamma$ of the Riccati equation (9) is positive semi-definite. If, in addition, the pair $(\bar{Q}, \bar{A})$ is observable, then $\boldsymbol{\Gamma}>\mathbf{0}$.

Lemma 6 (Knobloch [4]). Suppose $\overline{\boldsymbol{R}} \geqslant \mathbf{0}$ and $\boldsymbol{\Gamma}^{-}$is the solution of (9) making all eigenvalues of $\overline{\boldsymbol{A}}+\overline{\boldsymbol{R}} \boldsymbol{\Gamma}^{-}$ belong to the OLF'P. Let $\overline{\boldsymbol{\Gamma}}$ be any matrix satisfying (5), then $\Gamma^{-} \leqslant \overline{\boldsymbol{\Gamma}}$.

Summarizing the above three lemmas, and assuming a positive semi-definite $\boldsymbol{Q}$ is chosen, we obtain a set of sufficient conditions on $p$ and $r$ for the existence of $\Gamma>0$ to constitute a set of pi-coefficients.

Theorem 2. The inequality (5) has all positive-definite solutions if

(i) $\overline{\boldsymbol{R}} \geqslant \mathbf{0}$ and $\overline{\boldsymbol{Q}} \geqslant \mathbf{0}$,

(ii) $\bar{A}$ has all eigenvalues in $O L H P$,

(iii) $\boldsymbol{H}$ has no eigenvalues on the imaginary axis,

(iv) $(\bar{A}, \bar{R})$ is controllable and $(\bar{Q}, \bar{A})$ is observable.

Obviously, under the previous condition $M_{4}=-d+r+p d^{2}<0$, we have $\overline{\boldsymbol{R}} \geqslant \mathbf{0}$. If in addition $p r-\frac{1}{4} \leqslant 0$ is required, then $\bar{Q} \geqslant \mathbf{0}$. These two inequalities on $p$ and $r$, together with the assumption of controllability and observability of $\{\boldsymbol{A}, \boldsymbol{b}, \boldsymbol{c}\}$, imply condition (iv), as can be seen from the forms of $\overline{\boldsymbol{A}}, \overline{\boldsymbol{R}}$, and $\overline{\boldsymbol{Q}}$ in (6), (7), and (8), respectively.

Focusing on condition (ii), we see that $\bar{A}$ can be re-written as

$$
\tilde{\boldsymbol{A}}=\boldsymbol{A}-\left(-d+r+p d^{2}\right)^{-1}\left(p d-\frac{1}{2}\right) \boldsymbol{b} \boldsymbol{c}^{\mathrm{T}}=\boldsymbol{A}-\boldsymbol{k} \boldsymbol{b} \boldsymbol{c}^{\mathrm{T}} .
$$

Thus, $\bar{A}$ can be viewed as the state matrix of a fictitious LTI system described by $\{\boldsymbol{A}, \boldsymbol{b}, \boldsymbol{c}\}$ and controlled by a negative output feedback gain $k$. The range of $k$ within which the fictitious "closed-loop system" is stable can be determined by the following lemma.

Lemma 7 (Vidyasagar [8]). Let $g(s)=\boldsymbol{c}^{\mathrm{T}}(s \boldsymbol{I}-\boldsymbol{A})^{-1} \boldsymbol{b}$ and $h(s)=g(s) /[1+k g(s)]$. Suppose $\mu_{+}$is the number of poles of $g(s)$ with positive real part. Then $h(s)$ is stable if and only if

$$
1+k g(\mathrm{j} \omega) \neq 0, \forall \omega \in \mathbb{R} \cup\{ \pm \infty\} \quad \text { and } \quad \lim _{\omega \rightarrow \infty}[\phi(\mathrm{j} \omega)-\phi(-\mathrm{j} \omega)]=2 \pi \mu_{+}
$$

where $\phi(\mathrm{j} \omega)$ is the phase of $g(\mathrm{j} \omega)$.

Suppose from the Nyquist plot of $g(\mathrm{j} \omega)$, we can determine a range of $k, k_{-}<k<k_{+}$, within which $h(s)$ is stable. Since $k=\left(-d+r+p d^{2}\right)^{-1}\left(p d-\frac{1}{2}\right)$ and $-d+r+p d^{2}<0$, we conclude that $\bar{A}$ has all eigenvalues in OLHP if

$$
p d\left(k_{-} d-1\right)>k_{-}(d-r)-\frac{1}{2} \quad \text { and } \quad p d\left(k_{+} d-1\right)<k_{+}(d-r)-\frac{1}{2} .
$$


Next, conditions of $\boldsymbol{H}$ having no eigenvalues on the imaginary axis are developed. The Hamiltonian matrix $\boldsymbol{H}$ is defined in (10), and its characteristic polynomial is

$$
\begin{aligned}
\operatorname{det}(s \boldsymbol{I}-\boldsymbol{H}) & =\operatorname{det}\left[\begin{array}{cc}
s \boldsymbol{I}-\overline{\boldsymbol{A}} & -\overline{\boldsymbol{R}} \\
\overline{\boldsymbol{Q}} & s \boldsymbol{I}+\overline{\boldsymbol{A}}^{\mathrm{T}}
\end{array}\right] \\
& =\operatorname{det}(s \boldsymbol{I}-\overline{\boldsymbol{A}}) \operatorname{det}\left[s \boldsymbol{I}+\overline{\boldsymbol{A}}^{\mathrm{T}}+\overline{\boldsymbol{Q}}(s \boldsymbol{I}-\overline{\boldsymbol{A}})^{-1} \overline{\boldsymbol{R}}\right] \\
& =\operatorname{det}(s \boldsymbol{I}-\overline{\boldsymbol{A}}) \operatorname{det}\left(s \boldsymbol{I}+\overline{\boldsymbol{A}}^{\mathrm{T}}\right) \operatorname{det}\left[\boldsymbol{I}+\left(s \boldsymbol{I}+\overline{\boldsymbol{A}}^{\mathrm{T}}\right)^{-1} \overline{\boldsymbol{Q}}(s \boldsymbol{I}-\overline{\boldsymbol{A}})^{-1} \overline{\boldsymbol{R}}\right] \\
& =\operatorname{det}(s \boldsymbol{I}-\overline{\boldsymbol{A}}) \operatorname{det}\left(s \boldsymbol{I}+\overline{\boldsymbol{A}}^{\mathrm{T}}\right) Y(s) .
\end{aligned}
$$

For simplicity, we choose $\boldsymbol{Q}=\mathbf{0}$. Substituting $\overline{\boldsymbol{A}}, \overline{\boldsymbol{R}}$ and $\overline{\boldsymbol{Q}}$ into $Y(s)$, we have

$$
\begin{aligned}
Y(s)= & \operatorname{det}\left\{\boldsymbol{I}-\left[s \boldsymbol{I}+\boldsymbol{A}^{\mathrm{T}}-\left(-d+r+p d^{2}\right)^{-1}\left(p d-\frac{1}{2}\right) \boldsymbol{c} \boldsymbol{b}^{\mathrm{T}}\right]^{-1}\left[\left(p r-\frac{1}{4}\right)\left(-d+r+p d^{2}\right)^{-1} \boldsymbol{c c}^{\mathrm{T}}\right]\right. \\
& \left.\times\left[s \boldsymbol{I}-\boldsymbol{A}+\left(-d+r+p d^{2}\right)^{-1}\left(p d-\frac{1}{2}\right) \boldsymbol{b} \boldsymbol{c}^{\mathrm{T}}\right]^{-1}\left(-d+r+p d^{2}\right)^{-1} \boldsymbol{b} \boldsymbol{b}^{\mathrm{T}}\right\} .
\end{aligned}
$$

By the matrix inversion lemma and properties of determinants, (13) can be re-written as

$$
\begin{aligned}
Y(s)= & 1-\left(p r-\frac{1}{4}\right)\left(-d+r+p d^{2}\right)^{-2}\left[\frac{\boldsymbol{b}^{\mathrm{T}}\left(s \boldsymbol{I}+\boldsymbol{A}^{\mathrm{T}}\right)^{-1} \boldsymbol{c}}{1-\left(-d+r+p d^{2}\right)^{-1}\left(p d-\frac{1}{2}\right) \boldsymbol{b}^{\mathrm{T}}\left(s \boldsymbol{I}+\boldsymbol{A}^{\mathrm{T}}\right)^{-1} \boldsymbol{c}}\right] \\
& \times\left[\frac{\boldsymbol{c}^{\mathrm{T}}(s \boldsymbol{I}-\boldsymbol{A})^{-1} \boldsymbol{b}}{1+\left(-d+r+p d^{2}\right)^{-1}\left(p d-\frac{1}{2}\right) \boldsymbol{c}^{\mathrm{T}}(s \boldsymbol{I}-\boldsymbol{A})^{-1} \boldsymbol{b}}\right] .
\end{aligned}
$$

Again let $g(s)=\boldsymbol{c}^{\mathrm{T}}(s \boldsymbol{I}-\boldsymbol{A})^{-1} \boldsymbol{b}$ and $k=\left(-d+r+p d^{2}\right)^{-1}\left(p d-\frac{1}{2}\right)$, then we have

$$
Y(s)=\frac{\left(-d+r+p d^{2}\right)+\left(p d-\frac{1}{2}\right)(g(-s)+g(s))+p g(-s) g(s)}{\left(-d+r+p d^{2}\right)(1+k g(-s))(1+k g(s))} .
$$

Thus $\operatorname{det}(\mathrm{j} \omega \boldsymbol{I}-\boldsymbol{H})=\operatorname{det}(\mathrm{j} \omega \boldsymbol{I}-\overline{\boldsymbol{A}}) \operatorname{det}\left(\mathrm{j} \omega \boldsymbol{I}+\overline{\boldsymbol{A}}^{\mathrm{T}}\right) Y(\mathrm{j} \omega)$.

Suppose $\overline{\boldsymbol{A}}$ is guaranteed to have all eigenvalues in OLHP, so j $\omega$ will not be an eigenvalue of $\bar{A}$. Hence $\mathrm{j} \omega$ is an eigenvalue of $\boldsymbol{H}$ if and only if

$$
Y(\mathrm{j} \omega)=\frac{\left(-d+r+p d^{2}\right)+\left(p d-\frac{1}{2}\right)(g(-\mathrm{j} \omega)+g(\mathrm{j} \omega))+p g(-\mathrm{j} \omega) g(\mathrm{j} \omega)}{\left(-d+r+p d^{2}\right)(1+k g(-\mathrm{j} \omega))(1+k g(\mathrm{j} \omega))}=0 .
$$

By conditions developed above, we know that the denominator of (14) is nonzero, so the condition of $\boldsymbol{H}$ having eigenvalues on the imaginary axis is

$$
\left(-d+r+p d^{2}\right)+\left(p d-\frac{1}{2}\right)(g(-\mathrm{j} \omega)+g(\mathrm{j} \omega))+p g(-\mathrm{j} \omega) g(\mathrm{j} \omega)=0
$$

for some $\omega \in \mathbb{R}$. If notation $G(s)=\boldsymbol{c}^{\mathrm{T}}(s \boldsymbol{I}-\boldsymbol{A})^{-1} \boldsymbol{b}+\boldsymbol{d}$ is used, then (15) can be simplified to

$$
p=\frac{\operatorname{Re}[G(\mathrm{j} \omega)]-r}{|G(\mathrm{j} \omega)|^{2}}
$$

Therefore, if $\boldsymbol{H}$ is not to have eigenvalue on the imaginary axis, then (16) should not be satisfied, which means that on the $r-p$ plane, all lines corresponding to (16) for all $\omega \in \mathbb{R}$ should be rejected. As it is difficult to use the above formula directly, we develop the lemma below. 
Lemma 8. Let

$$
\begin{array}{ll}
k_{1}=\inf _{\omega} \operatorname{Re}[G(\mathrm{j} \omega)], & k_{2}=\inf _{\omega} \frac{\operatorname{Re}[G(\mathrm{j} \omega)]}{|G(\mathrm{j} \omega)|^{2}}, \\
k_{3}=\inf _{\omega}|G(\mathrm{j} \omega)|^{2}, & k_{4}=\sup _{\omega}|G(\mathrm{j} \omega)|^{2} .
\end{array}
$$

If $k_{1}>0$, then the lines of (16) are bounded below by the following three lines:

$$
\begin{aligned}
& p=-\frac{1}{k_{4}} r+k_{2} \quad \text { for } r<0, \\
& p=-\frac{k_{2}}{k_{1}} r+k_{2} \quad \text { for } 0 \leqslant r \leqslant k_{1}, \\
& p=-\frac{1}{k_{3}} r+\frac{k_{1}}{k_{3}} \text { for } r>k_{1} .
\end{aligned}
$$

Proof. First we note that if $k_{1}>0$, then all $k_{i}>0$ for $i=1, \ldots, 4$. For $r<0$, comparing (16) with (17), we get

$$
\frac{\operatorname{Re}[G(\mathrm{j} \omega)]-r}{|G(\mathrm{j} \omega)|^{2}}-\left(-\frac{1}{k_{4}} r+k_{2}\right)=\left(\frac{\operatorname{Re}[G(\mathrm{j} \omega)]}{|G(\mathrm{j} \omega)|^{2}}-k_{2}\right)-r\left(\frac{1}{|G(\mathrm{j} \omega)|^{2}}-\frac{1}{k_{4}}\right) \geqslant 0 .
$$

For $0 \leqslant r \leqslant k_{1}$, comparing (16) with (18), we get

$$
\begin{aligned}
& \frac{\operatorname{Re}[G(\mathrm{j} \omega)]-r}{|G(\mathrm{j} \omega)|^{2}}-\left(-\frac{k_{2}}{k_{1}} r+k_{2}\right)=\left(\frac{\operatorname{Re}[G(\mathrm{j} \omega)]}{|G(\mathrm{j} \omega)|^{2}}-k_{2}\right)-\frac{r}{k_{1}}\left(\frac{k_{1}}{|G(\mathrm{j} \omega)|^{2}}-k_{2}\right) \\
& \geqslant\left(\frac{\operatorname{Re}[G(\mathrm{j} \omega)]}{|G(\mathrm{j} \omega)|^{2}}-k_{2}\right)-\frac{r}{k_{1}}\left(\frac{\operatorname{Re}[G(\mathrm{j} \omega)]}{|G(\mathrm{j} \omega)|^{2}}-k_{2}\right)=\left(\frac{\operatorname{Re}[G(\mathrm{j} \omega)]}{|G(\mathrm{j} \omega)|^{2}}-k_{2}\right)\left(1-\frac{r}{k_{1}}\right) \geqslant 0 .
\end{aligned}
$$

For $r>k_{1}$, comparing (16) with (19), we get

$$
\begin{aligned}
& \frac{\operatorname{Re}[G(\mathrm{j} \omega)]-r}{|G(\mathrm{j} \omega)|^{2}}-\left(-\frac{1}{k_{3}} r+\frac{k_{1}}{k_{3}}\right)=\left(\frac{\operatorname{Re}[G(\mathrm{j} \omega)]}{|G(\mathrm{j} \omega)|^{2}}-\frac{k_{1}}{k_{3}}\right)+r\left(\frac{1}{k_{3}}-\frac{1}{|G(\mathrm{j} \omega)|^{2}}\right) \\
& \geqslant\left(\frac{k_{1}}{|G(\mathrm{j} \omega)|^{2}}-\frac{k_{1}}{k_{3}}\right)+r\left(\frac{1}{k_{3}}-\frac{1}{|G(\mathrm{j} \omega)|^{2}}\right)=\left(r-k_{1}\right)\left(\frac{1}{k_{3}}-\frac{1}{|G(\mathrm{j} \omega)|^{2}}\right) \geqslant 0 .
\end{aligned}
$$

It is noted that for the cases in which $k_{1} \leqslant 0$, similar approach can be used to derive formulas of lines forming a boundary of a region not passed through by lines of (16). This region on the $r-p$ plane, when intersected with the regions described by $-d+r+p d^{2}<0, p r-\frac{1}{4}<0$, and (12) gives a feasible region of the pi-coefficients $r$ and $p$.

\section{An example}

Consider the feedback system shown in Fig. 1, in which the forward path contains an LTI system $S_{1}$ with transfer function:

$$
G(s)=\frac{(s+2)(s+6)}{(s+1)(s+3)(s+4)}+0.1,
$$

and the feedback path contains a relay $S_{2}$ which has a hysteresis characteristic shown in Fig. 2 and described by

$$
\psi(\sigma)=\left\{\begin{array}{cl}
b & \text { if } \sigma \geqslant a, \text { or }|\sigma|<a \text { and } \sigma \text { enters this region from } \sigma \geqslant a, \\
-b & \text { if } \sigma \leqslant-a, \text { or }|\sigma|<a \text { and } \sigma \text { enters this region from } \sigma \leqslant-a
\end{array}\right.
$$




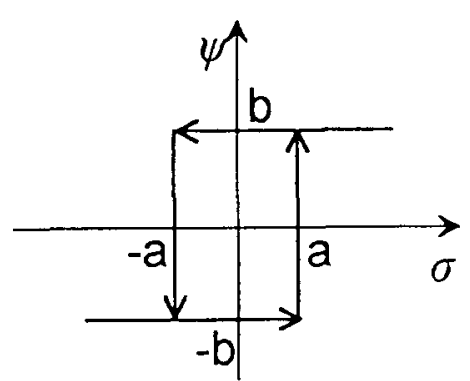

Fig. 2. Relay characteristic.

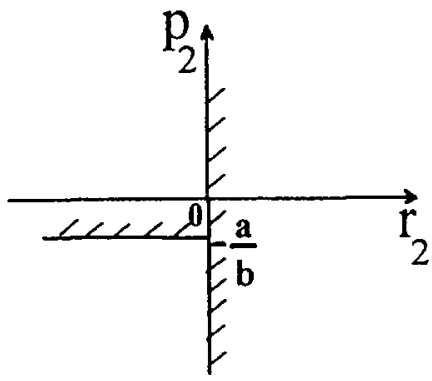

Fig. 4. Feasible region of $S_{2}$

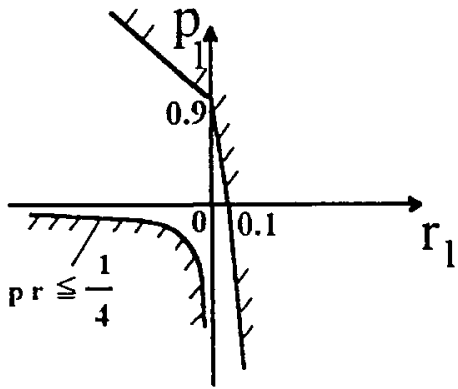

Fig. 3. Feasible region of $S_{1}$.

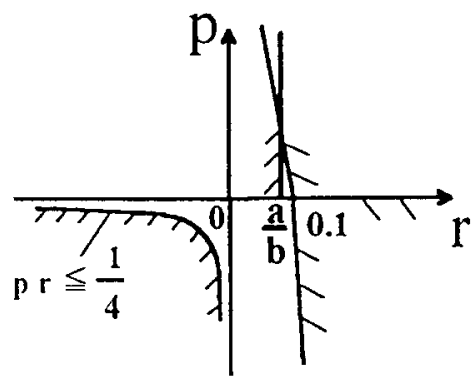

Fig. 5. Stability condition.

where $\psi$ and $\sigma$ are the output and input of the relay, respectively. The objective is to determine the stability condition for this feedback system.

Although in this case the feedback system is nonlinear, Theorem 1 is still applicable. The reason is that pi-sharing theory is developed for nonlinear systems [7]. In fact, the form of (1) allows the consideration of nonlinear system if we let $\boldsymbol{A}(t), \boldsymbol{b}(t), \boldsymbol{c}(t), d(t)$ depend on $\boldsymbol{x}(t)$ and $u(t)$ [7]. Also note that the nonlinearity $\psi$ has no states $\boldsymbol{x}(t)$, so its pi-coefficients degenerate to $p$ and $r$ only. Consequently if the LTI subsystem in the forward path has a positive-definite $\Gamma$, then we only need to ensure that condition (ii) of Theorem 1 holds.

By using the results developed in the above section, a feasible region on the $r-p$ plane can be constructed for $S_{1}$ after relevant constants $k_{-}, k_{+}$, and $k_{1}, \ldots, k_{4}$, are determined: $k_{-}=-1, k_{+}=\infty, k_{1}=0.1, k_{2}=0.9091$, $k_{3}=0.0101$ and $k_{4}=1.2099$. A graphical representation of the feasible region is shown in Fig. 3. Corresponding to each point in the region, a $\Gamma>0$ exists for $S_{1}$. As for the relay $S_{2}$, the derivation of a feasible set of $p$ and $r$ can be found in the Appendix, and the corresponding $r-p$ diagram is shown in Fig. 4.

Thus, we know that the feedback system is pi-stable if $p_{1}+r_{2}>0$ and $r_{1}+p_{2}>0$. To check these conditions, we can draw the feasible region of one subsystem and the mirror image of that of the other subsystem with respect to the line $p+r=0$ on the same $r-p$ plane as shown in Fig. 5. If these two regions have an overlap area, then the conclusion is affirmative. Using this method, we can determine that for $a<0.1 b$, the feedback system is pi-stable, or the system has bounded outputs and bounded states for all bounded inputs.

\section{Conclusions}

In this paper we discuss the pi-sharing theory for continuous-time systems. For LTI state-space models we develop an analytic approach for computing time-invariant pi-coefficients. The results are useful in the determination of stability of feedback systems. In the example provided we show an application involving linear and nonlinear systems simultaneously. It is believed that for certain more complicated nonlinear systems, such 
as those from adaptive control systems, pi-coefficients can also be found. Hence, the stability of combination of such systems with LTI systems can be studied. Currently, researches in this direction are underway.

\section{Appendix}

Consider a relay with the hysteresis characteristic described by (21). By the definition of pi-coefficients and the fact that there are no states involved, the system constituted by this nonlinearity is pi-sharing with respect to constant pi-coefficients $\{p, r\}$ if

$$
\int_{0}^{T} \sigma(t) \psi(t) \mathrm{d} t \geqslant \int_{0}^{T} p \psi^{2}(t) \mathrm{d} t+\int_{0}^{T} r \sigma^{2}(t) \mathrm{d} t .
$$

The above inequality holds if for all $t$ we have

$$
\sigma(t) \psi(t)-p \psi^{2}(t)-r \sigma^{2}(t) \geqslant 0 .
$$

Consider the part with $|\sigma| \geqslant a$ first. Since $\psi^{2}=b^{2}$ and $\sigma \psi=b|\sigma|$ now, (A.2) can be re-written as

$$
b|\sigma|-p b^{2}-r|\sigma|^{2} \geqslant 0 \text {. }
$$

Obviously, if (A.3) is to hold for all $|\sigma| \geqslant a$, then it is impossible to let $r>0$. For $r=0$, (A.3) reduces to $|\sigma| \geqslant p b$, which holds for all $|\sigma| \geqslant a$ when $p \leqslant a / b$. For $r<0$, if the quadratic function of $|\sigma|$ in (A.3) has nonnegative value and derivative at $|\sigma|=a$, then (A.3) is satisfied for all $|\sigma| \geqslant a$. Thus for $r<0$

$$
p \leqslant \frac{a b-a^{2} r}{b^{2}}
$$

and $r \leqslant b / 2 a$ are enough to guarantee (A.3) for all $|\sigma| \geqslant a$. In summary, for $|\sigma| \geqslant a$ feasible pairs of $\{p, r\}$ must satisfy $r \leqslant 0$ and (A.4).

Next consider the part with $|\sigma|<a$. Now we have $\psi^{2}=b^{2}$ and $\sigma \psi \geqslant-a b$. Hence a sufficient condition for (A.2) is

$$
-a b-p b^{2}-r \sigma^{2} \geqslant 0
$$

Because the possibility of having $r>0$ has been ruled out in the previous case, we need to consider $r \leqslant 0$ only. For (A.5) to hold under current constraints, clearly, we can only choose

$$
p \leqslant-\frac{a}{b}
$$

Combining these results, we know a feasible region of pi-coefficients on the $r-p$ plane is described by (A.6) and $r \leqslant 0$.

\section{References}

[1] S. Boyd, L. El Ghaoui, E. Feron, V. Balakrishnan, Linear Matrix Inequalities in System and Control Theory, SIAM, Philadelphia, 1994.

[2] J.-S. Heh, I-K. Fong, J.-F. Wu, Pi-coefficient analysis of update algorithms for adaptive systems, Systems Control Lett. 20 (1993) $289-297$.

[3] J.-S. Heh, J.-F. Wu, I-K. Fong, About the computation of optimal pi-coefficients, Proc. American Control Conf., 1992, pp. $1063-1064$.

[4] H.W. Knobloch, A. Isidori, D. Flockerzi, Topics in Control Theory, Birkhauser, Basel, 1993.

[5] E. Kreindler, A. Jameson, Conditions for nonnegativeness of partitioned matrices, IEEE Trans. Automat. Control 17 (1972) $147-148$.

[6] D.A. Lawrence, A.daptive system stability analysis via energy exchange, Ph.D. dissertation, Cornell University, 1985.

[7] D.A. Lawrence, C.R. Johnson, Jr., Recursive parameter identification algorithm stability analysis via pi-sharing, IEEE Trans. Automat. Control 31 (1986) 14-24.

[8] M. Vidyasagar, Nonlinear Systems Analysis, Prentice-Hall, Englewood Cliffs, NJ, 1993.

[9] J.-F. Wu, J.-S. Hєh, I-K. Fong, On computing pi-coefficients, Systems \& Control Lett. 16 (1991) 457-463. 